Canadian Oncology

Nursing Journal

Revue canadienne

de soins infirmiers

en oncologie

Volume 28, Issue 1 • Winter 2018

elSSN: 2368-8076 


\title{
Autonomiser les patients et les soignants grâce au savoir : Élaboration d'une formation sur la chimiothérapie en gynécologie oncologique dirigée par le personnel infirmier
}

\author{
par Nazlin Jivraj, Lisa Ould Gallagher, Janet Papadakos, Nazek Abdelmutti, Aileen Trang et Sarah E. Ferguson

\section{RÉSUMÉ}

L'augmentation du nombre de patients vus en clinique dans un centre de cancérologie du Canada est venue nuire à l'offre d'un enseignement de qualité sur la chimiothérapie pour les patients et leur famille. Le département de gynéco-oncologie a cerné plusieurs obstacles à la transmission d'un enseignement complet et opportun. Une fois le diagnostic de cancer tombé, l'accès à l'enseignement, l'efficacité de l'information transmise par écrit, les contraintes de temps des infirmières et l'absence d'uniformité dans la prise en charge des effets secondaires figurent parmi les obstacles relevés. Une équipe interdisciplinaire s'est donc rassemblée pour revoir les pratiques d'enseignement actuelles et se pencher sur le programme d'enseignement aux patients afin de développer, conjointement, des stratégies pour régler ces problèmes. Cet article décrit les étapes ayant mené à l'élaboration, par le personnel infirmier, d'une formation sur la chimiothérapie (protocoles courants) destinée aux patients atteints de cancers gynécologiques et visant à aider les patients et les soignants à mieux se préparer aux séances de chimiothérapie, à calmer l'anxiété et à savoir comment gérer les effets secondaires liés au traitement.

\section{AU SUJET DES AUTEURES}

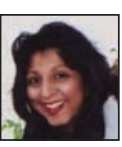

Nazlin Jivraj, inf. aut., B.Sc.Inf., CSIO(C), infirmière spécialisée en oncologie, soins ambulatoires en gynéco-oncologie, Centre de cancérologie Princess Margaret, Toronto, ON.

Courriel : nazlin.jivraj@uhn.ca (auteure-ressource)

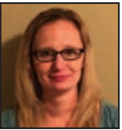

Lisa Ould Gallagher, inf. aut., CSIO(C), infirmière spécialisée en oncologie, soins ambulatoires en gynéco-oncologie, Centre de cancérologie Princess Margaret, Toronto, $\mathrm{ON}$.

Courriel : lisa.gallagher@uhn.ca

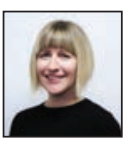

Janet Papadakos, M.Ed., Ph.D. (c), codirectrice, Centre de recherche sur la littératie en santé et cancer, Senior Manager, Recherche sur l'enseignement relatif au cancer, Centre de cancérologie Princess Margaret, Provincial Head, Patient Education, Cancer Care Ontario. Toronto, ON. Courriel : Janet.Papadakos@uhnresearch.ca

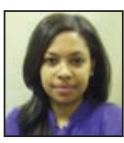

Nazek Abdelmutti, M.Sc.(Htlh Communication), Manager, Cancer Strategy Stewardship, Centre de cancérologie Princess Margaret, Toronto, ON.

Courriel : Nazek.abdelmutti@uhnresearch.ca

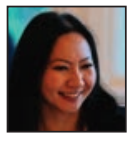

Aileen Trang, M.Sc., Division d'oncologie-hématologie médicale, Groupe des essais cliniques, Centre de cancérologie Princess Margaret, Toronto, ON. Courriel : aileen.trang@uhn.ca

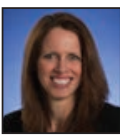

Sarah E. Ferguson, MD, FRCSC : Division de gynéco-oncologie, Réseau universitaire de santé (University Health Network), Département d'obstétrique et de gynécologie, Université de Toronto, ON. Courriel : sarah.ferguson@uhn.ca

DOI: $10.5737 / 23688076281812$

\section{INTRODUCTION}

T a Société canadienne du cancer (2015) estime qu'enviـron 196900 Canadiens ont reçu un diagnostic de cancer en 2015. Parmi ces personnes, quelque 10508 étaient touchées par un cancer gynécologique. Ces taux d'incidence ont été relevés dans notre centre de cancérologie. En 2011, le centre de cancérologie a rencontré près de 700 patients à qui on venait de diagnostiquer un cancer gynécologique, comparativement à 494 en 2001 (Registre du cancer, 2016). Cette hausse de patients, dans un contexte de soins où les demandes d'enseignement sont nombreuses et le temps, limité, est venue compromettre la capacité des infirmières à s'investir auprès des patients et à leur prodiguer l'enseignement nécessaire en consultation externe.

Or, les protocoles de traitements utilisés pour les cancers gynécologiques comprennent de la chimiothérapie, seule ou combinée à de la radiothérapie - des traitements complexes qui exigent beaucoup des patients. L'observance des protocoles, entre autres, est plus difficile sans avoir reçu une bonne formation et préparation. Les infirmières certifiées en oncologie détiennent l'expertise requise pour transmettre les connaissances nécessaires aux patients et aux soignants (Association canadienne des infirmières en oncologie (CANO/ACIO), 2006), car leur pratique est guidée par les normes établies par l'ACIO. Les normes de pratique de l'ACIO mandatent les infirmières spécialisées en oncologie pour transmettre aux patients et aux soignants l'information relative aux protocoles de traitement, à la gestion des effets secondaires et aux stratégies d'adaptation (CANO/ACIO, 2006).

Dans les cliniques gynécologiques, les infirmières éprouvaient des difficultés à transmettre un enseignement complet aux patients en rai son des contraintes de temps doublées de la forte anxiété des patients ayant reçu un diagnostic récent et des craintes associées au début de la chimiothérapie (Askren, 2013; Dalby et al., 2013; Sheldon, 2008). La problématique se traduisait par un nombre croissant d'appels téléphoniques de patientes aux infirmières en oncologie au sujet des effets secondaires, de la prise d'antiémétiques, des séances de chimiothérapie ou des raisons justifiant une visite aux urgences. Les préoccupations grandissantes des patients combinées à l'augmentation de leur nombre affectaient directement la capacité des infirmières à s'investir dans un enseignement global au patient et dans sa prise en charge. Une formation sur la chimiothérapie en gynécologie oncologique, dirigée par des infirmières, a donc été conçue pour remédier à la situation. 
Dans cet article, les auteures présentent les étapes de développement de la formation, le programme d'enseignement, ainsi que l'apport du programme.

\section{CONTEXTE}

Plusieurs publications ont fait état des obstacles reliés à la prestation de soins et à l'enseignement relatif au traitement prodigué aux patients atteints de cancer (Askren, 2013; Chelf et al., 2001; Dalby et al., 2013; Malone, 2007; Portz et Johnston, 2014; Williams et Schreier, 2004). Parmi ces obstacles, notons : la difficulté à trouver le moment opportun pour prodiguer l'enseignement au patient une fois le diagnostic tombé (Malone, 2007; Williams et Schreier, 2004), l'efficacité du matériel écrit (Williams et Schreier, 2004; Chelf et al. 2001; Dalby et al., 2013), les contraintes de temps auxquelles les infirmières font face (Askren, 2013), et le manque d'uniformité dans l'approche de prise en charge des effets secondaires (Portz et Johnston, 2014). Ces obstacles rapportés dans la littérature reflètent ceux auxquels se butent les infirmières de notre centre de cancérologie.

Malone (2007) ainsi que Williams et Schreier (2004) signalent que les patients ressentent de l'anxiété au moment où ils apprennent qu'ils ont un cancer et qu'ils subiront une chimiothérapie. Ils peuvent se sentir facilement dépassés par les événements et incapables de comprendre ou retenir l'information en lien avec les effets secondaires et la routine associés à la chimiothérapie. Lorsque cela se produit, les patients se rendent inutilement aux urgences (Mann, 2011). Ils peuvent aussi ne pas reconnaitre les symptômes dangereux potentiels et consulter une fois ces symptômes exacerbés (Schofield, 2008; D'Amato, 2008), au risque d'interrompre le plan de traitement et de nuire à son efficacité (D’Amato, 2008).

Dans notre centre de cancérologie, les patients rencontrés en consultation externe sont systématiquement informés de leur diagnostic et de leur plan de traitement, qu'il s'agisse d'une chirurgie gynécologique ou d'une procédure de diagnostic. Les infirmières en oncologie ont remarqué l'incapacité de plusieurs patients à retenir dans ce contexte l'information concernant le début des traitements de chimiothérapie. Les patients appellent à plusieurs reprises après avoir reçu leur première dose de chimiothérapie afin d'obtenir (de nouveau) des explications sur les effets secondaires, les médicaments et le calendrier de chimiothérapie.

Divers éléments affectent l'expérience des patients atteints de cancer : l'important sentiment d'anxiété et de bouleversement, associé à la capacité du patient à assimiler des informations au sujet du cancer, en plus du matériel d'enseignement souvent rédigé dans un niveau de lecture bien plus avancé que celui rencontré chez la plupart des patients (Portz et Johnston, 2014; Williams et Schreier, 2004; Chelf et al., 2001; Dalby et al., 2013). Les infirmières utilisent régulièrement des feuillets d'information sur la chimiothérapie pour revoir les effets secondaires du traitement avec le patient et le soignant. Cependant, selon Williams et Schreier (2004), ces outils d'enseignement ne sont pas souvent rédigés dans un niveau de lecture accessible à tous. Plusieurs auteurs font remarquer que ce matériel devrait être écrit en langage clair et correspondant à un niveau de lecture de Ge année ou moins, puisque les patients n'ont pas tous les mêmes capacités de lecture (Chelf et al., 2001; Dalby et al., 2013; Doak, Doak et Root, 1996).

Le manque de temps constitue un autre obstacle de taille dans la prestation d'enseignement au patient qui reçoit des traitements de chimiothérapie. Une enquête menée par Askren (2013) a indiqué que les infirmières étaient assaillies par de nombreuses demandes au travail et que cela affectait considérablement le temps consacré à l'enseignement au patient. Askren (2013) a rapporté l'incapacité des infirmières à compléter l'enseignement au patient, et cela en raison du nombre très élevé de patients à voir, au fait que plus d'une heure soit nécessaire pour transmettre l'enseignement aux patients n'ayant jamais eu d'expérience liée à la chimiothérapie, et que les patients se sentent dépassés par l'excès d'information reçue. S'ensuivit une évaluation plus poussée des patients et des soignants concernant la pertinence et l'utilité de l'information transmise. Ce sondage a révélé que l'enseignement donné sur la gestion des symptômes était considéré comme insuffisant (Askren, 2013), ce qui peut entraîner une mauvaise prise en charge des symptômes, de hauts niveaux d'anxiété et une incapacité à faire face à la situation vécue (McCaughan et McKenna, 2007).

Le manque de temps pour l'enseignement aux patients était devenu habituel pour les infirmières en gynéco-oncologie en consultation externe, et le temps pris pour transmettre aux patients et soignants l'information relative à la chimiothérapie affectait les autres tâches infirmières dans la clinique. Ces pressions temporelles affectaient la productivité de l'enseignement, plus encore lorsque les patients étaient déjà angoissés. Bien souvent, les infirmières devaient fournir l'enseignement à deux et même trois patients n'ayant jamais reçu de chimiothérapie en plus de s'occuper d'une clinique de vingtcinq patients, tout cela en trois heures et demie.

Les infirmières en gynéco-oncologie ont également souligné que la prise en charge de certains effets secondaires n'était pas uniforme. De fait, chaque infirmière utilisait sa propre méthode pour soulager les effets secondaires, occasionnant de la confusion chez les patients qui demandaient de l'assistance. L'importance d'une approche standardisée lors d'une première séance d'information sur la chimiothérapie a été présentée dans les travaux de Dalby et collègues (2013). Léquipe de Dalby s'est mobilisée pour améliorer les soins aux patients et la satisfaction du personnel avec l'utilisation d'une liste de contrôle, de matériel d'éducation et de méthodes d'enseignement standardisés.

Afin de régler ces problèmes, les infirmières en gynéco-oncologie ont collaboré avec le programme d'éducation aux patients afin d'explorer comment tirer parti des programmes d'enseignement existant déjà à l'interne. L'un de ces programmes consistait en une formation générale sur la chimiothérapie destinée aux patients et aux soignants. Le contenu de cette formation était large et développé pour des patients touchés par différents types de cancers et traités par toutes sortes de protocoles de chimiothérapie. Une évaluation de cette formation en 2011 a montré qu'elle amenait une baisse d'anxiété des patients, mais une hausse chez les soignants 
(Papadakos, 2010). Dans leurs réponses aux questions ouvertes de l'évaluation, les patients et les soignants expliquaient que le programme de formation couvrait trop large, et qu'il serait préférable d'obtenir un enseignement adapté au type de cancer et aux protocoles de chimiothérapie qui s'y appliquent. Ils ont également signalé que les renseignements fournis étaient trop vastes et souvent non applicables à leur situation (Papadakos, 2010). Il a donc été décidé de développer une formation sur la chimiothérapie pour les cancers gynécologiques.

\section{Élaboration d'un programme sur mesure}

Afin de justifier la mise en place d'un enseignement adapté à la chimiothérapie gynécologique, les infirmières ont consulté les données provenant du système d'information sur les patients en oncologie (OPIS) au centre de cancérologie. Selon ces données, environ 250 patients auraient reçu un nouveau régime thérapeutique en 2010 (OPIS, 2010). Ces résultats avaient entraîné des analyses plus poussées en 2011 : chaque infirmière en gynéco-oncologie a alors compilé les paramètres de sa pratique personnelle en recensant pendant un mois le nombre de nouvelles séances d'enseignement données aux patients, le temps consacré à cet enseignement, et le nombre d'appels téléphoniques subséquents se rapportant à la chimiothérapie (effets secondaires, médication, calendriers, etc.).

On a ainsi pu constater qu'en avril 2011, 37 patients avaient commencé un nouveau protocole de traitement. En se basant sur le temps consacré à l'enseignement aux patients rapporté par les infirmières, on a évalué que la durée moyenne passée avec chaque patient était de vingt minutes. La charge de travail était divisée entre une infirmière en gynéco-oncologie à temps partiel et six autres à temps complet. Lorsque la langue était une barrière et nécessitait la présence d'un interprète, l'enseignement sur la chimiothérapie était beaucoup plus long.

La rencontre de 20 minutes par patient a été jugée insuffisante, compte tenu du nombre d'appels passés pour obtenir des précisions. Les registres des appels téléphoniques des patients ont été étudiés, pour révéler que plusieurs de ces requêtes quotidiennes visaient à obtenir des clarifications sur ce qui avait été enseigné durant la séance de 20 minutes. En avril 2011, les infirmières ont noté les paramètres des types d'appels suivants : effets secondaires, médication et calendriers de traitement. Sur les 168 appels faits, 139 avaient trait à la gestion des symptômes, 6 à la prise des médicaments et 23 à l'horaire des rendez-vous. La nature des appels téléphoniques a indiqué que la plupart des patients ne se souvenaient pas des informations données en lien avec la gestion des symptômes, la médication et la logique des rendez-vous attribués. Près de 83 \% des appels étaient associés au contrôle des symptômes (questions à propos de nausées, vomissements, constipation, douleurs osseuses, fièvre ou neuropathies, surtout).

Les conclusions ont été présentées au groupe de gynéco-oncologie, avec la proposition d'élaborer une formation sur la chimiothérapie en gynécologie oncologique visant à améliorer les connaissances des patients et des soignants pour pouvoir diminuer le nombre d'appels téléphoniques destinés aux infirmières. Le personnel infirmier et médical s'est entendu sur les protocoles courants de chimiothérapie à enseigner et l'uniformisation de la prise en charge des effets secondaires. Un consensus a été atteint pour prioriser les trois protocoles les plus communément proposés aux patients ayant un cancer gynécologique : Carboplatine, Paclitaxel et Cisplatine. Les médecins ou les infirmières devaient identifier tous les patients n'ayant jamais reçu de traitement de chimiothérapie, qui assisteraient à la séance d'information une semaine avant d'amorcer leur traitement. Les patients ont été obligatoirement inscrits à cette séance préalable sur la chimio.

Il a été décidé que l'enseignement serait assuré par une infirmière spécialisée en oncologie et possédant une expertise en gynéco-oncologie, au sein de petits groupes réunissant des patients et des soignants (Keller, 2006). Le programme d'enseignement se concentre sur quatre objectifs : à quoi s'attendre pendant les visites, comment gérer les effets secondaires, comment gérer ses émotions, et comment la famille et les amis peuvent aider.

En plus d'une formation d'une heure, des guides protocolaires de chimiothérapie ont été développés. Ces guides ont été élaborés en utilisant un niveau de lecture de $6^{\mathrm{e}}$ année afin d'accroître la rétention des informations transmises pendant la séance. Par exemple, le guide renseigne sur la façon de prendre des antiémétiques et sur les raisons devant pousser à contacter une infirmière en oncologie ou à se rendre aux urgences (Malone, 2007; Dalby et al., 2013). Les infirmières en gynéco-oncologie ont collaboré avec les oncologues ainsi que le programme d'éducation aux patients afin de créer ces ressources ainsi que la présentation de type PowerPoint utilisée pendant la formation.

\section{RÉSULTATS}

De janvier 2012 à décembre 2015, 538 patients et 506 soignants ont assisté à la formation. De plus, plusieurs membres du personnel (infirmières, coordonnateurs de rendez-vous, pharmaciens, travailleurs sociaux, infirmières gestionnaires et autres membres du corps médical) se sont joints occasionnellement à la classe. Bien qu'aucune mesure d'évaluation n'ait été prise à proprement parler, les infirmières ont observé depuis la mise en place de la formation une diminution marquée du nombre d'appels en lien avec les médicaments utilisés pour la chimiothérapie, les effets secondaires et les rendez-vous. Nous pensons que cela prouve bien que le temps consacré à l'éducation des patients et des soignants à propos des traitements, lors d'une rencontre prévue à cet effet, a amélioré la gestion du temps en consultation externe et permet d'offrir des soins mieux personnalisés pour ces patients. Les évaluations complétées par les patients et les soignants à la fin de chaque formation le montrent puisque de nombreux témoignages de patients reconnaissent la nécessité de ce projet mené par le corps infirmier.

- « Le cours est une très bonne idée. C'est mieux que de seulement recevoir des documents... Savoir à quoi s'attendre est plus sécurisant que de ne pas le savoir. En comprenant que je pourrai obtenir toute l'aide et les conseils nécessaires pendant cette épreuve, je me sens rassurée. »

- « L'information transmise a été très utile, elle m’a aidée à 
surmonter l'inconnu et mes peurs. »

- «Atmosphère détendue et rassurante. La formatrice m’a fait me sentir à l'aise pour poser mes questions. »

- Les témoignages des soignants allaient dans le même sens :

- «Les formatrices ont fourni des éléments très instructifs et ont répondu aux questions. En tant que membre de la famille, cela m'a aidé à répondre à certaines questions qui me tracassaient. Je suis content d'avoir assisté à cette séance. »

- « Je pense qu'il est très important que le soignant puisse prendre des notes pour le patient. Il y a beaucoup d'information à assimiler. L'animatrice était très renseignée et s'exprimait bien. »

- «Je crois qu'il est très bénéfique pour le patient d'être accompagné d'un membre de sa famille (une personne qui demeure avec lui). »

De ces évaluations ressort l'importance d'adapter l'information en fonction des différents groupes de patients. Pour ce faire, il fallait mieux développer le programme de formation afin que les protocoles de chimiothérapie soient traités séparément. Auparavant, tous les patients traités par chimiothérapie recevaient la même formation, sans égard au protocole de traitement qu'ils vivraient. Par exemple, une infirmière pouvait enseigner aux patients traités au Carboplatine et au Paclitaxel, ou qui subissaient un traitement concomitant de Cisplatine et de radiothérapie lors d'une seule et même rencontre. Les patients ont indiqué que cette méthode de transmission de l'information créait de la confusion chez eux. Les infirmières ont alors décidé d’offrir des formations séparées et spécifiques au protocole de traitement. Actuellement, trois formations sur la chimiothérapie d'une heure chacune sont données par le personnel infirmier :

- Chimiothérapie intraveineuse à base de Paclitaxel et de Carboplatine (une fois par semaine)

- Chimiothérapie intrapéritonéale à base de Cisplatine et de Paclitaxel (une fois par deux semaines)

- Traitement concomitant de radiothérapie et de chimiothérapie à base de Cisplatine (une fois par deux semaines)

Le groupe de gynéco-oncologie a fait en sorte que l'enseignement aux patients n'ayant jamais subi de chimiothérapie devienne une «norme de soins ». De plus, le nouveau personnel et les étudiants en soins infirmiers doivent obligatoirement assister à la formation, ce qui leur permet de comprendre l'information transmise aux patients et de prodiguer des soins uniformes. La formation portant sur l'oncologie gynécologique a créé un précédent au sein d'autres groupes du centre de

\section{RÉFÉRENCES}

Askren, H. (2013). Patient binders: Creating a tool that helps patients and nurses. Retrieved from http://www.oncologynurseadvisor.com/ survivorship/patient-binders-creating-a-tool-that-helps-patientsand-nurses/article/310253/

Canadian Association of Nurses in Oncology/Association canadienne des infirmières en oncologie (CANO $\backslash$ ACIO). (2006). Practice Standards and Competencies for the Specialized Oncology Nurse (CANO/ACIO). Retrieved from http://www.cano-acio.ca cancérologie, les amenant à uniformiser leur approche d'enseignement auprès de leurs différents groupes de patients.

\section{NOUVELLES INITIATIVES}

Avec le succès retentissant de la formation, plusieurs nouvelles initiatives sont considérées pour continuer à améliorer le concept. Nombre de ces initiatives se fondent sur les rétroactions des patients et des soignants. Les projets suivants sont examinés :

- Créer des séries vidéo pour faire ressortir ce qui caractérise les autosoins lors d'une chimiothérapie

- Rendre tout le matériel disponible sur Internet pour les patients et les soignants

- Traduire toutes les ressources dans les principales langues parlées au centre de cancérologie

- Élaborer des guides de protocoles chimiothérapeutiques pour les différents types de cancers gynécologiques

- Concevoir une trousse d'informations à l'intention des soignants axée sur le « Stress des soignants »

- Développer une version en ligne de la formation afin d'uniformiser l'enseignement et d'en faciliter l'accès aux patients qui demeurent à l'extérieur de la ville

- Développer une version en ligne de la formation pour le personnel afin d'uniformiser l'enseignement offert aux patients et à leurs soignants.

\section{CONCLUSION}

La formation sur la chimiothérapie en gynécologie oncologique dans notre centre de cancérologie a permis d'offrir une approche éducative uniforme aux patients qui reçoivent leur premier traitement de chimiothérapie en consultation externe. Ce projet, mené par le personnel infirmier, a permis d'établir une trajectoire d'autonomisation des patients et des soignants qui, avec de nouvelles connaissances et une confiance personnelle renouvelée, peuvent ressentir moins d'anxiété pendant le traitement. La formation sur la chimiothérapie, donnée par des infirmières, est conforme aux normes de pratique de l'ACIO en ce qui a trait à la relation thérapeutique basée sur le soutien, à la gestion des symptômes du cancer, aux effets secondaires du traitement et à l'enseignement et à l'encadrement (ACIO, 2006). Depuis le début de la formation, les infirmières ont affirmé recevoir moins d'appels téléphoniques au sujet de la chimiothérapie. Par ailleurs, la création de cette formation fournit une plateforme pour concevoir d'autres programmes d'enseignement axés sur différents sièges de la maladie, et pouvant être offertes de diverses façons.

Canadian Cancer Society's Advisory Committee on Cancer Statistics. (2015). Canadian Cancer Statistic 2015. Toronto, ON: Canadian Cancer Society. [May 2015, adapted June 2015] ISSN 0835-2976

Chelf, J.H., Agre, P., Axelrod, A., Cheney, L., Cole, D.D., Conrad, K., $\&$ Weaver, C. (2001). Cancer-related patient education: An overview of the last decade of evaluation and research. Oncology Nursing Forum, 28(7), 1139-1147. 
Dalby, C.K., Nesbitt, M., Frechette, C.A., Kennerley, K., Lacoursiere, L.H., \& Buswell, L. (2013). Standardization of initial chemotherapy teaching to improve care. Clinical Journal of Oncology Nursing, 17(5), 472-475. doi:10.1188/13.CJON.472-475

D'Amato, S. (2008). Improving patient adherence with oral chemotherapy. Oncology Issues, 42-45.

Doak, C.C., Doak, L.G., \& Root, J.H. (1996). Teaching patients with low literacy skills. Philadelphia: J.B. Lippincott Company.

Keller, J. (2006). Implementation of a prechemotherapy educational intervention for women newly diagnosed with breast cancer. Clinical Journal of Oncology Nursing, 10(1), 57-63. doi:10.1188/06. CJON.57-60

Malone, P. (2007). Implementation of a prechemotherapy educational intervention. Clinical Journal of Oncology Nursing, 11(5), 707-710. doi:10.1188/07.CJON.707-710

Mann, K. (2011). Education and health promotion for new patients with cancer: A quality improvement model. Clinical Journal of Oncology Nursing, 15(1), 55-61. doi:10.1188/11.CJON.55-61

McCaughan, E., \& McKenna, H. (2007). Never-ending making sense: Towards a substantive theory of the information-seeking behaviour of newly diagnosed cancer patients. Journal of Clinical Nursing, 16(11), 2096-2104. doi:10.1111/j.1365-2702.2006.01817x

Oncology Patient Information System (OPIS) at Princess Margaret Cancer Centre (2010). Toronto, Ontario.
Papadakos, J., Brecher, E., Seki, J., Lee, R., Stewart, J., Abdelmutti, N. (2010, October). Effect of the PMH 'Chemo Questions?' class on anxiety and self-efficacy in cancer patients and caregivers: A summative evaluation. International Cancer Patient Education Meeting San Diego, California.

Portz, D., \& Johnston, M.P. (2014). Implementation of an evidencebased education practice change for patients with cancer. Clinical Journal of Oncology Nursing, 18(5), 36-40. doi:10.1188/14.CJON. S2.36-40

Princess Margaret Cancer Registry (2016). New primary gynecological cancer cases seen at Princess Margaret 2001-2015. Princess Margaret Cancer Centre. Toronto, Ontario.

Schofield, P., Jefford, M., Carey, M., Thomson, K., Evans, M., Baravelli, C., \& Aranda, S. (2008). Preparing patients for threatening medical treatments: Effects of a chemotherapy educational DVD on anxiety, unmet needs, and selfefficacy. Supportive Care in Cancer, 16(1), 37-45. doi:10.1007/ s00520-007-0273-4

Sheldon, L.K., Swanson, S., Dolce, A., Marsh, K., \& Summers, J. (2008). Putting evidence into practice: Evidence-based interventions for anxiety. Clinical Journal of Oncology Nursing, 12(5), 789-797. doi:10.1188/08.cjon.789-797

Williams, S., \& Schreier, A. (2004). The effect of education in managing side effects in women receiving chemotherapy for treatment of breast cancer. Oncology Nursing Forum, 31(1),16-23. doi:10.1188/04.ONF.E16-E23 